Luo, T, Gao, F \& Hoff, K. S. (2013). Examining student participation in three learning activities supported by social annotation tools. Journal of Educational Technology Development and Exchange, 6(2), 53-68

\title{
Examining Student Participation in Three Learning Activities Supported by Social Annotation Tools
}

\author{
Tian Luo \\ Ohio University \\ Fei Gao \\ Bowling Green State University \\ Kathryn S Hoff \\ Bowling Green State University
}

\begin{abstract}
Social annotation (SA) allows learners to highlight and comment on Web pages and share annotations with each other online. Despite its potential in promoting collaborative learning, examining how to integrate it into educational settings has not been fully studied. The purpose of the study is to examine student participation in three different SA-based online activities: (1) peer review, (2) annotated discussion, and (3) collaborative reading. Students participated in all three SA-based activities and took a survey at the end reporting the effectiveness of these activities. The analysis of students' annotations and their survey responses suggested that although participants perceived the use of SA tools in all the three activities to be relatively effective, their levels of participation in the three activities varied greatly. The authors discuss the pros and cons of using SA in each of the activities based on the findings. Suggestions for future use and development of SA tools are also provided.
\end{abstract}

Keywords: social annotation, collaboration, peer review, annotated discussion, collaborative reading

\section{Introduction}

Social annotation (SA) tools are one type of online application that enables multiple learners to annotate and comment collaboratively on content material synchronously or asynchronously on the Web. In contrast to traditional text-based annotations that are hard to share among learners, social annotation tools allow learners to work continuously and collaboratively on one file, and the annotations and comments are automatically stored in an online database for everyone to review (Novak, Razzouk, \& Johnson, 2012). Essentially, social annotation tools promote collaboration by eliminating the back-and-forth information exchange process and enabling real-time learner-centered collaborative annotation (Nokelainen et al., 2005; Su, Yang, Hwang, \& Zhang, 2010). 
In recent years, the number of studies conducted examining the adoption of social annotation tools in educational settings in an attempt to discover what effect they have on the achievement of students has been increasing (Novak et al., 2012). Despite the enthusiasm in using SA tools for teaching and learning, relevant research in this area is still limited. In our study, the authors attempt to explore possible ways of using SA tools to support collaborative learning in online learning settings by examining three types of SA-supported instructional activities in a graduate-level online course, and reporting students' perceived learning experiences and the challenges of using SA tools educationally.

\section{Research on SA-Supported Collaborative Learning}

SA tools are an emerging technology that has not yet been widely used or investigated in education, and related research is still scarce (Novak et al., 2012). Nevertheless, due to the social and collaborative nature of SA tools, there has been research conducted to examine the incorporation of SA tools to support collaborative learning (Glover, Xu, \& Hardaker, 2007; Hwang, Wang, \& Sharples, 2007; Johnson, Archibald, \& Tenenbaum, 2010; Nokelainen et al., 2005; Samuel, Kim, \& Johnson, 2011). In this section is a review of the research concerning SA tools being used in educational settings and their effects on learning.

\subsection{Educational Use of SA Tools}

Despite potential that the use of SA tools has to promote collaborate learning, research in this specific area has been minimal. In fact, the majority of extant studies pertaining to collaborative learning were conducted in online environments such as discussion forums, wikis, and blogs (Yadegaridehkordi, Iahad, \& Ahmad,
2013). Contrastingly, SA tools were rarely examined. In reviewing the empirical studies on the use of SA tools, the authors found that reading comprehension and peer review appear to be the two major activities in which SA tools were used and evaluated.

\subsubsection{Reading comprehension.} Depending on how the instructor sets up the collaborative learning activity, the integration of social annotation tools could take different forms. One popular use is to engage learners in reading comprehension activities (Johnson et al., 2010; Kawasaki, Sasaki, Yamaguchi, \& Yamaguchi, 2008; Razon, Turner, Johnson, Arsal, \& Tenenbaum, 2012; Sakar \& Ercetin, 2005; Samuel et al., 2011). In such activities, learners are instructed to read learning materials available on the Web or provided by the instructor. Using a social annotation platform, learners can interact with learning materials and each other by adding markups and annotations as the material is being read.

The degree of collaboration varies significantly in those reading comprehension activities. In some studies, minimal collaboration was involved as the purpose of these activities was primarily to enhance individual learners' reading comprehension skills (Kawasaki et al., 2008; Sakar \& Ercetin, 2005; Samuel et al., 2011). Learners used more traditional and fundamental features in SA tools such as comments and markups to facilitate their individual reading processes. Those features allowed learners to anchor within the context, making annotations associated with particular words, paragraphs of a text, or a section of an entire document (Wolfe, 2002).

Some studies place more emphasis on the collaborative aspect of learning (Hwang et al., 2007; Johnson et al., 2010; Mendenhall, 
2010; Nokelainen et al., 2005). The use of SA tools in such environments encourages both synchronous and asynchronous collaboration among learners and instructors. When multiple learners are working on the same reading material using SA tools synchronously, they can comment on the text and engage in real-time discussion. Collaboration can also be achieved asynchronously as learners work on the same file at their own pace. Learners will receive real-time notifications as annotations are created. The instructor can design activities that allow collaborations to take place both synchronously in classroom settings and asynchronously outside the classroom, depending on the learning goals and the degree of flexibility the instructor would like to provide (Novak et al., 2012).

2.1.2. Peer review. Peer review is a type of collaborative activity commonly used in the classroom where instructors ask the students to write their reflections, share with one another, and critique each other's work in small groups (Mendenhall, 2010; Samuel et al., 2011; van der Pol, van den Berg, Admiraal, \& Simons, 2008). SA tools afford peer review activities by providing a convenient platform in which interactions among peers can be seamlessly accomplished.

Similar to collaborative reading activities, peer review with SA tools can occur synchronously and asynchronously to support student learning. In one study (Mendenhall, 2010), students were grouped into pairs providing feedback to one another. The feedback could be either general (one that is not linked to a specific portion of the text) or specific. Mendenhall (2010) asserted that the use of SA tools allowed students to focus on specific portions of the text during the critiquing process. However, research that examines SA-supported peer review activities remains limited.

\subsection{Educational Effects}

There is evidence to suggest that participation in SA-supported collaborative learning activities can impact student learning affectively and cognitively. In the affective learning domain, it has been found that using SA tools may enhance student engagement, participation, and motivation. Across the studies, the majority of students perceived the use of SA tools favorably (Kawasaki et al., 2008; Mendenhall, 2010; Nokelainen et al., 2005; Novak et al., 2012). Researchers also found that students were highly motivated when they participated in SA-supported activities (Hwang et al., 2007; Kawasaki et al., 2008; Nokelainen et al., 2005; Razon et al., 2012; Samuel et al., 2011).

In the cognitive learning domain, studies were conducted to investigate if SA-supported activities promote student learning. Some experimental and quasi-experimental studies have suggested that reading comprehension skills can be potentially enhanced by SAsupported reading activities (Johnson et al., 2010; Kawasaki et al., 2008; Mendenhall, 2010; Razon et al., 2012). In using SA tools, students reported that they were more likely to stay on task and more focused on the reading material. Researchers found that when working in small groups or participating in peer review activities, students were able to investigate their own mental models through interacting with their peers, allowing them to engage in a deep and reflective thought process (Merrill \& Gilbert, 2008). Some stated that SA tools facilitated the group/pair work process by reducing the redundancy of critique items and promoting the construction of concentrated and in-depth content (Mendenhall, 2010). Others reported that annotations promoted more reflective responses to a text (Wolfe, 2008). However, researchers have not found any statistically significant results when measuring such variables as critical thinking 
and meta-cognitive skills (Johnson et al., 2010; Mendenhall, 2010; Razon et al., 2012).

A brief review of current research indicates that SA tools have the potential to support collaborative learning. As Novak et al. (2012) noted more research needs to be done in this field given the fact that the use of this technology in general is still in its embryonic stage. As demonstrated in the literature review, to date, SA tools in most of the research studies are used for reading comprehension purposes (Novak et al., 2012). Few studies have explored the use of SA tools for purposes other than enhancing student reading comprehension. The authors failed to find any studies in which the adoption of SA tools was accomplished in a fully online environment. Questions such as how instructors should use SA tools to help students learn more effectively, and how to design such activities in a purely online learning environment remain unaddressed.
In this study, the authors examined three distinct SA-supported activities in an online course. We showcased the activities that we designed and implemented for unique educational purposes, and addressed the following research questions by analyzing data collected from the survey, along with students' online annotations and markups. We used the following research questions to guide the research:

1)How did students participate in the three collaborative SA-supported activities?

2) How did students perceive the effectiveness of the SA-supported activities?

3) What were students' perceived challenges and limitations of integrating SA tools into teaching and learning?

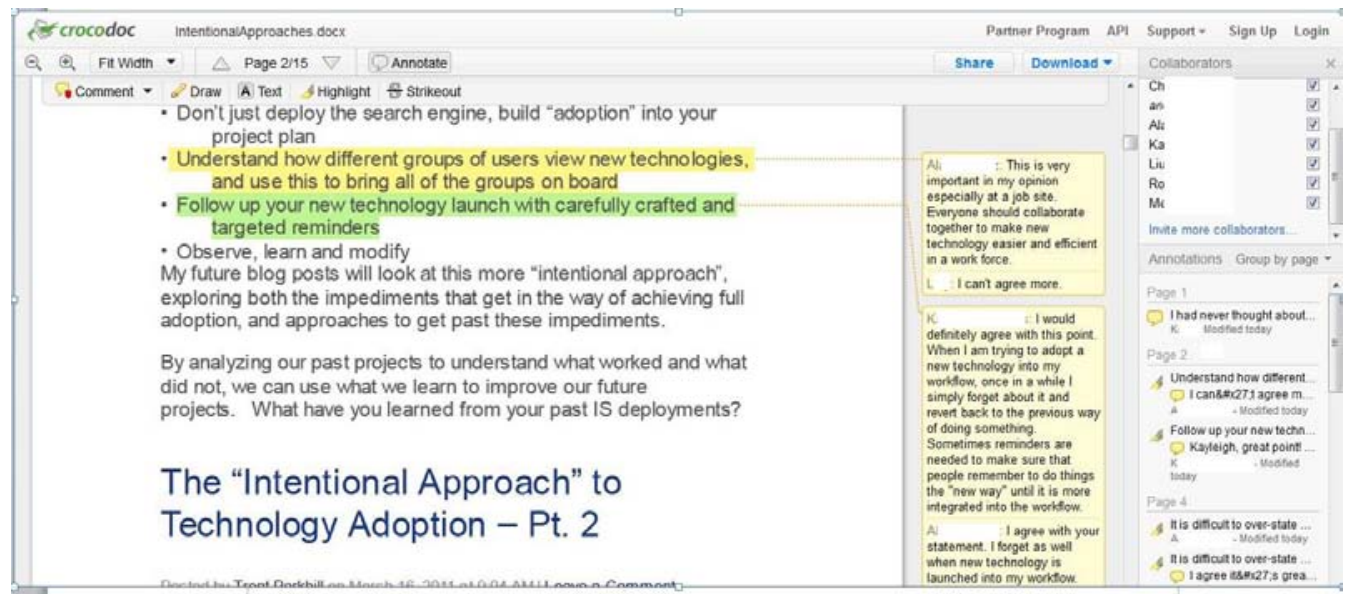

Figure 1. Screenshot of the crocodoc interface 
3. Two Social Annotation Environments: Crocodoc and Wikispaces

\subsection{Crocodoc}

As a collaborative Web annotation tool, Crocodoc supports basic annotation features such as highlights, sticky notes, strikeouts, and collaborative functions that allow users to add and reply to comments on a shared document in real time. New annotations are displayed simultaneously to other users who are viewing the file concurrently (see Figure 1). Offline users can also receive real-time notifications when annotations are created.

\subsection{Wikispaces}

The online course was held on Wikispaces. The built-in annotation tool worked as one of the collaborative editing functions afforded by Wikispaces. Once registered and logged in students could edit the same page collaboratively with other online users. In the editing mode, the annotation tool in Wikispaces allows users to highlight one part of the text and add sticky notes on the side. Users need to save their changes in the editing mode so that other users will be able to see it afterwards. The annotated page shows the comments, the time when the comment is created, and the comment creator's name (see Figure 2).

\section{Method}

\subsection{Research Design}

The adopted methodological approach is a case study. Case studies embrace a multidimensional approach to analysis, especially through the use of multiple sources of evidence (Yin, 2009). A combination of qualitative and quantitative methods is often found in case studies and serves the best purposes, as the different strengths and weaknesses of both qualitative and quantitative methods are essentially complementary.

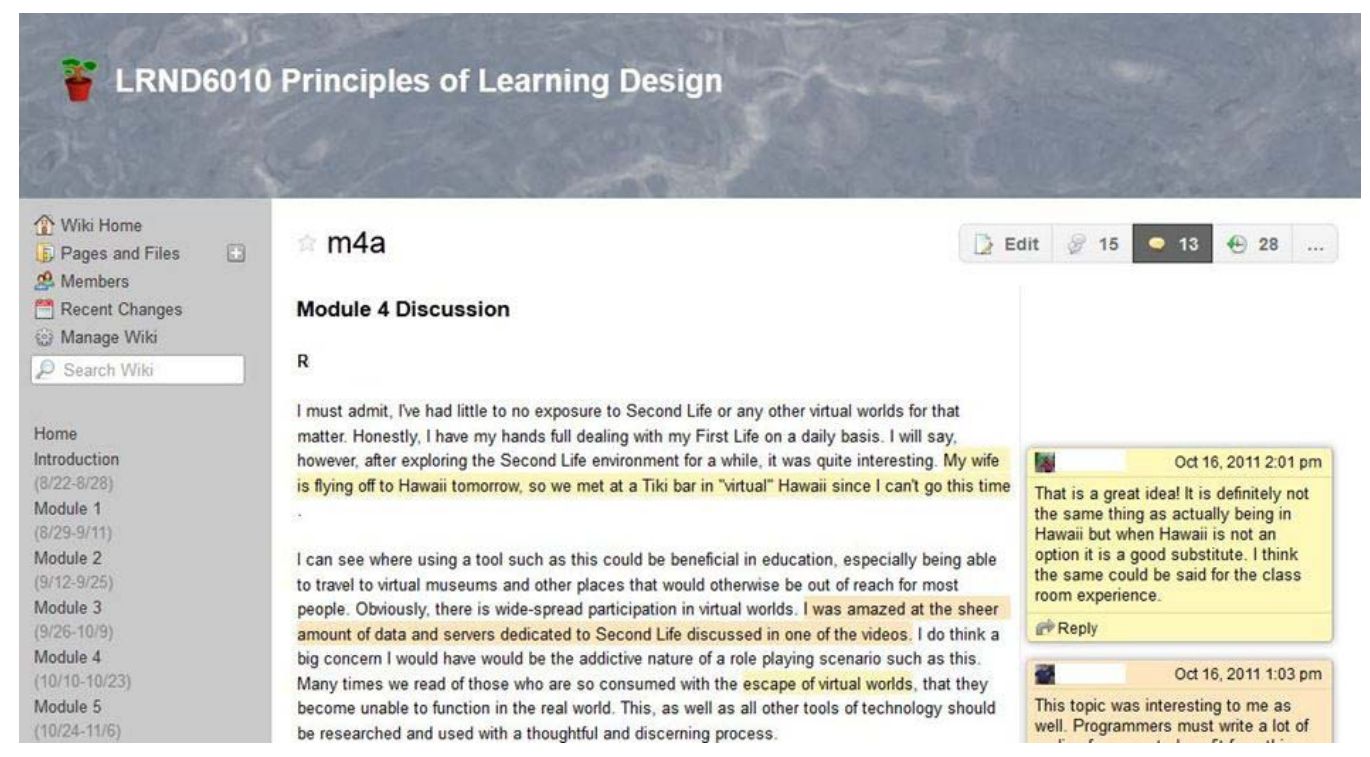

Figure 2. Demo of the annotation tool within Wikispaces interface 
In this study, three collaborative SAsupported instructional activities were selected and examined in-depth to answer the research questions. Both qualitative and quantitative data were collected, including formative data generated from the three collaborative activities and student responses to a survey at the end of the course. The survey consists of three sections: (1) questions on students' overall enjoyment of using SA tools in this course, (2) questions on students' perceived effectiveness of using SA tools in the three collaborative activities, and (3) students' demographic information.

\subsection{Participants and Settings}

Participants were seven graduate students taking an online class on Instructional Design. The average age of this group is 38 , among which three were between 20 to 30 years old, two between 31 to 50, and two above 50. Most participants reported to possess a high level of technological literacy. All of them have had experience taking online courses prior to this course (see Table 1).
The goal of this online course was to explore and synthesize relevant literature in the learning field, explain how technology impacts and influences learning, evaluate the effectiveness of learning technologies, and experiment with technology-supported teaching and learning strategies. On the Wikispaces course site, students were provided with tutorials that introduced the SA tools used in this course. Because online discussion and collaboration were important parts of the course, students were expected to visit the course site at least three times a week for effective learning.

\subsection{SA-Supported Instructional Activities}

Because this course is purely online, activities supported by collaborative online tools provided important means for students to interact with and learn from each other. Students participated in three unique SAsupported instructional activities throughout the semester. All of them were graded as a part of the assessments of the course; that is, student participation in these activities was required, not optional.

Table 1. Participants profile

\begin{tabular}{lllc}
\hline Name & $\begin{array}{l}\text { Self-rated technological } \\
\text { literacy level }\end{array}$ & Age & Number of online courses taken \\
\hline Candy & Beginner & 49 & 2 \\
Emmy & Intermediate & 24 & 1 \\
Kathy & Advanced & 26 & 3 \\
Alex & Advanced & 34 & 2 \\
Melissa & Advanced & 58 & 7 \\
Jennifer & Advanced & 23 & 4 \\
Roy & Expert & 52 & 1 \\
\hline
\end{tabular}


4.3.1. Instructional activity 1: peer review. This peer review activity was embedded in a module on reflective learning to engage students in a reflective learning process. Students posted their lesson plans online in Crocodoc for others to review and were instructed to provide feedback to two of their peers' lesson plans. The instructor suggested two ways of providing comments: (1) using the social annotation tools in Crocodoc to provide specific comments, and (2) writing more general and summative comments at the bottom of the lesson plan page using a different color. Students were not required to use the annotation function, but it was encouraged. The instructor also provided detailed guidelines on how to conduct peer review to ensure the quality of the comments.

\subsubsection{Instructional activity 2: annotated} discussion. In the module - virtual learning environments, students were required to use the annotation/commenting function embedded in Wikispaces to discuss with their classmates on a wiki page on their experience with Second Life and the educational potential and challenges of virtual worlds. The rationale behind this activity is to highlight annotation functions that allow students to respond directly to specific sentences or paragraphs of their classmates' posts, which is not possible in most online discussion forums. Students were asked to post at least two replies to others' posts using the annotation function and were free to decide the length of their replies.

4.3.3. Instructional activity 3: collaborative reading. The third activity was a collaborative reading activity in Crocodoc. An article on technology adoption was shared in Crocodoc. Students were instructed to share their ideas and critiques while reading the article online by making at least one comment and responding to at least one of their classmates' comments. The purpose of this activity was to have students construct a collective and thorough understanding of the article by reading and annotating the article collaboratively.

\subsection{Data Analysis}

The data analyzed in this study included students' annotations and comments and the summative survey responses. The data sources were triangulated to better answer the research questions.

As the three activities were conducted at different times with varying purposes, the authors chose to analyze the data generated from each activity separately. The number of annotations/comments, as well as the number of words in each annotation/comment, was counted. Also the quality of the comments was examined to understand student participation. Student-perceived effectiveness of the SA tools was measured by calculating the mean and standard deviations of student ratings on their perception of each activity in the survey. Finally, responses to the open-ended questions in the survey were coded to identify the perceived challenges and limitations of using SA tools in education.

\section{Results}

\subsection{Student Participation}

5.1.1. Instructional activity 1. In the first collaborative activity, few participants used SA tools to complete the peer review tasks, as their use was optional rather than mandatory. Most reviewers just posted summative comments, and only two used the annotation features to provide specific feedback by highlighting and focusing on particular sections of the lesson plans. Table 2 demonstrates student participation in this activity.

In contrast to the majority of learners, Kathy and Alex demonstrated great interest 
Table 2. Student participation in peer review

\begin{tabular}{|c|c|c|c|}
\hline ID & Name & $\begin{array}{l}\text { Provided summative } \\
\text { review }\end{array}$ & Provided in-text annotation \\
\hline 1 & Kathy & Yes & Yes \\
\hline 2 & Alex & Yes & Yes \\
\hline 3 & Emmy & Yes & No \\
\hline 4 & Roy & Yes & No \\
\hline 5 & Candy & Yes & No \\
\hline 6 & Melissa & Yes & No \\
\hline 7 & Jennifer & Yes & No \\
\hline
\end{tabular}

in using the SA tools to provide specific content-pertinent feedback. For example, Kathy provided 20 responding comments in total to two peers' lesson plans, including two summative comments and 18 specific comments using the SA tool. The feedback focused on the specifics in the lesson plans and involved detailed suggestions, probing questions, and useful resources. In one of Kathy's annotations, for example, she highlighted and commented on the implementation procedures of her peer's lesson plan, stating that "I found these steps to be a little bit confusing. I think that is because there was not enough detail." She further suggested her peer utilize what was learned from the class to revise his lesson plan:

Now that we have read over Merrill's first principles, I think that your lesson plan would adapt well to these principles. You may want to consider restructuring these steps so that they follow Merrill's first principles exactly: Introduce the Problem, Activation, Demonstration, Application, Integration. You had addressed the first two steps and the last step to some extent in the other steps; however, I think that it would help to clearly identify each step along the way and provide a bit more detail as to the content of each step.

5.1.2. Instructional activity 2. In the discussion activity, all seven students wrote responses in the wiki site to the instructor's prompting questions, and five students engaged in collaborative discussion using the SA tool in Wikispaces and provided 15 total comments on the first four posts. On average, each student provided approximately two comments (mean=2.14, $\mathrm{SD}=1.57$ ). The average length of the posts was approximately 112 words. Looking at the 15 comments contributed by seven participants, we found that Alex, Roy, and Melissa were responsible for 11 comments. Other students, such as Kathy and Jennifer, chose only to provide one or two long and elaborative comments. One student, Candy, chose to respond only to instructor's questions. Student participation in this activity is demonstrated in detail in Table 3.

5.1.3. Instructional activity 3. In this activity, all seven participants engaged in the collaborative reading on Crocodoc and 
Table 3. Student participation in collaborative discussion

\begin{tabular}{llccc}
\hline ID & Name & $\begin{array}{l}\text { Number of words of } \\
\text { each student's post in } \\
\text { response to instructor's } \\
\text { questions }\end{array}$ & $\begin{array}{l}\text { Number of } \\
\text { replies to their } \\
\text { classmates' posts } \\
\text { per student }\end{array}$ & $\begin{array}{l}\text { Average number } \\
\text { of words of the } \\
\text { replies }\end{array}$ \\
\hline 1 & Kathy & 458 & 2 & 91 \\
2 & Alex & 524 & 3 & 46 \\
3 & Emmy & 394 & 1 & 29 \\
4 & Roy & 210 & 4 & 26 \\
5 & Candy & 434 & 0 & 0 \\
6 & Melissa & 765 & 4 & 35.25 \\
7 & Jennifer & 508 & 1 & 73 \\
\hline
\end{tabular}

Table 4. Student participation in collaborative reading

\begin{tabular}{clcc}
\hline ID & Name & number of posts & average number of words (mean=45.07) \\
\hline 1 & Kathy & 6 & 42.33 \\
2 & Alex & 4 & 33 \\
3 & Emmy & 4 & 20.75 \\
4 & Roy & 2 & 34.5 \\
5 & Candy & 2 & 76 \\
6 & Melissa & 5 & 53.4 \\
7 & Jennifer & 2 & 55.5 \\
\hline
\end{tabular}

generated 26 annotations on the reading material using the SA tool. The average number of annotations per student was roughly four (mean $=3.71, \mathrm{SD}=1.70$ ). The average number of words in each post was 45.07 per student. Students who contributed more in the previous activity, such as Kathy, Alex, and Melissa, remained active in this activity. Although the overall participation using SA tools was higher, the issue of unequal level of participation remained unchanged. Student participation in this activity is presented in Table 4 .

\subsection{Perceived Effectiveness of SA-Supported Activities}

Table 5 displays the means and standard deviations of students' ratings of their perceived effectiveness of the SA-supported activities in each collaborative activity. 
Table 5. Student perceived effectiveness of using SA tools

\begin{tabular}{|c|c|c|c|c|c|c|}
\hline ID & Name & $\begin{array}{l}\text { The } \\
\text { annotation } \\
\text { tools } \\
\text { supported } \\
\text { effective } \\
\text { peer review }\end{array}$ & $\begin{array}{l}\text { The } \\
\text { annotation } \\
\text { tools } \\
\text { supported } \\
\text { effective } \\
\text { discussion }\end{array}$ & $\begin{array}{l}\text { The annotation } \\
\text { tools supported } \\
\text { effective } \\
\text { collaborative } \\
\text { reading }\end{array}$ & $\begin{array}{l}\text { Average } \\
\text { Ratings }\end{array}$ & $\begin{array}{l}\text { Standard } \\
\text { Deviation }\end{array}$ \\
\hline 1 & Kathy & 4 & 3 & 4 & 3.67 & .58 \\
\hline 2 & Alex & 2 & 2 & 3 & 2.33 & .58 \\
\hline 3 & Emmy & 2 & 3 & 3 & 2.67 & .58 \\
\hline 4 & Roy & 4 & 4 & 2 & 3.33 & 1.15 \\
\hline 5 & Candy & 3 & 3 & 3 & 3.00 & 0 \\
\hline 6 & Melissa & 3 & 3 & 3 & 3.00 & 0 \\
\hline 7 & Jennifer & 3 & 3 & 2 & 2.67 & .58 \\
\hline \multicolumn{2}{|c|}{$\begin{array}{l}\text { Mean and } \\
\text { SD of each } \\
\text { statement }\end{array}$} & $3.00(.82)$ & $3.00(.58)$ & $2.86(.69)$ & & \\
\hline
\end{tabular}

Overall, participants perceived the use of SA tools in the three activities to be relatively effective. Six out of seven participants rated their perceptions favorably. Only Alex reported a neutral perceived effectiveness. Four students reported that the tools were very easy to use and intuitive to master. As Jennifer wrote, the use of annotation tools was effective "because it allows for concise feedback in an easy to follow format. It makes it easy to see the content and the comments simultaneously in an organized manner."

Interestingly, students' ratings of their perceptions were not always consistent with their participation in the collaborative activities. Some students, such as Kathy and Melissa, who participated actively in all three activities, also rated the three activities highly. In contrast, Alex was among the most active participants of the class, but he rated the effectiveness of two activities as " 0 ." This is counter-intuitive, as the authors might naturally assume that students who participate more actively should rate the system more positively. A closer look at Alex's written comments showed that Alex, in general, preferred a synchronous mode of communication as compared to asynchronous collaborative annotation by saying, "Basic discussions would be fine to discuss on the annotation tool in Wikispaces," but "I feel we can get more in a live setting vs. what is said on Wikispaces."

An analysis of students' responses to open-ended questions also revealed their attitudes toward each of the activities. When asked about the peer review activity, participants reported that the use of SA tools 
was effective in associating the comments with the corresponding texts. Kathy wrote that "It is very helpful to know exactly where in the article the reviewer is addressing his or her comment." Alex also reported that "I thought it was very helpful to point out specific parts of the article." In addition, Roy reported that "It makes it easy to see the content and the comments simultaneously in an organized manner." In the discussion activity, students considered the way that the SA tool was effective in presenting and organizing comments. Four students stated that they benefited most from being able to pinpoint specific content and highlight the part of the document on which they were commenting. As Jennifer stated, these features made it easy for them to collaboratively comment on each other's work. In the collaborative reading activity, students were able to identify more affordances of SA tools in addition to those in the previous two activities. Melissa felt that "It was really great to have a conversation with peers over an article. I was very interested to read what they were thinking as they went through the article." Some other students reported that the collaborative reading helped them attend to some issues that they would have otherwise missed, "There were some things in the article that I glimpsed over before, and having their comments there, I paid more attention to it." Additionally, Jennifer believed that the use of SA tools allowed for ongoing discussion on the reading material.

\subsection{Challenges and Limitations}

The SA tools used in this study hold promise for fostering collaborative learning. Most students perceived this integration favorably and benefited from using those tools. However, challenges and limitations coexist with the benefits. From students' responses to the open-ended questions, the authors identified the following challenges that instructors need to be mindful of when attempting to incorporate SA tools.

\subsubsection{Technical glitches. Although} participants in this study were relatively technologically capable, some of them still encountered intermittent technical difficulties resulting from some immature functionalities. In particular, when multiple students were simultaneously working on the same file, students reported that some iterations were not updated in time. This hampered the ongoing communication and interaction during the collaborative learning process.

\subsubsection{Difficulty in affording sustained} conversation. In the discussion and peer review activities, participants raised the issue of the SA tools current capability in sustaining conversation. Because students were only able to post their annotations on the margins of a page, the comments became cumbersome and difficult to read when the conversation lengthened. It also added a layer of difficulty for people to truly build upon the ideas and contributions of others. As Alex stated, because the place where comments and annotations were displayed was restricted, the comments tended to be brief, and it was difficult to be engaged in "a true back and forth discussion."

\subsubsection{Difficulty in affording solid content} creation. Participants also noted that some comments generated through SA tools were relatively superficial. Although this is not necessarily a problem with SA tools, participants found that some comments were lacking depth, and were therefore, brief and superficial. As Melissa commented, "The comments you make and receive are brief and fairly superficial. The reviews I gave and received were not in-depth." It could be that SA tools tend to afford meaningful construction that is contextualized within specific content rather than conclusive and indepth comments. 


\section{Discussion}

Included in this discussion are the levels of student participation in the three SAsupported activities. These include examining peer review, discussion, and collaborative reading, the pros and cons of using SA in each of the activities, and suggestions for future practice and SA tool development.

First, the SA tools were used by only two of the seven students in the peer review activity. Although students acknowledged the benefits of using SA tools, participation was minimal, as students were not required to use the tools. As shown in the findings, most students only provided a summative comment in this activity instead of providing an intext comment using the SA tools. One reason may be that students were unfamiliar with the SA tools as it was their first experience using them in this class. The fact that a few students used the SA tools extensively suggested that although some students may choose not to use them, others may feel this was a particularly useful tool for their own learning. As a result, it could be important for instructors to provide options to students so they can select whatever tool works for them during the learning process. This study suggests that more instructional support may be necessary to enhance the effectiveness of using SA tools for peer review activities. If the instructor provides necessary tips and examples of what types of annotations are expected, continuously encourages interaction and probes for in-depth thinking, students may be more likely to engage in a deeper analysis of their peers' work (Johnson et al., 2010; Mendenhall, 2010; Razon et al., 2012).

The SA tools were used with relative effectiveness in fostering discussion. The authors found that students particularly favored SA tools because they support focused and to-the-point discussions. With SA tools, students can highlight a specific part of the text and make comments on the side margin that allows for to-the-point discussions (van der Pol et al, 2008). Through this method, students were more likely to focus on specific learning materials and associate knowledge construction with specific learning content (Wolfe, 2002). Additionally, students could concentrate and comment on one particular page at a time, which reduces the cognitive load involved and can be beneficial to learning (Sweller, 1988). However, sustained and elaborate discussion was restricted due to the limitations of SA tools. When a number of students have multiple conversations in the SA platform, some functionality of SA tools such as the way student comments are displayed may restrict the depth and length of the conversation. Therefore, an improved system is needed to enable threaded discussions anchored to specific sections of the page without visually overwhelming the page or margins would be ideal (Johnson, Archibald, $\&$ Tenenbaum, 2010).

Students' participation in collaborative reading was more active as compared to the previous two activities. Findings showed that the use of the SA tool in the collaborative reading activity enabled students to learn from each other's ideas and also directed their attention to important sections of the reading. Using SA tools, students can review and analyze reading materials in a more detailed fashion, highlight important information within the text, and have discussions with their peers on the reading materials (Nokelainen et al., 2005). The authors also found that the use of SA tools allowed for an ongoing discussion on the reading material. As students were continuously working on only one primary text, they were able to build upon the existing texts and comments. This finding suggests that the collaborative reading activity seemed to be 
most favored by students as compared to the other two activities. Given the current features afforded by SA tools, collaborative reading activity seems to be best afforded because SA allows learners to effectively analyze and review the reading materials.

The findings in this study suggest that the use of SA tools holds promise for fostering collaborative learning. However, promoting critical thinking and meta-cognitive learning reflected by in-depth discussion and sustained conversation remains an issue. In this study, students responded positively to the use of the SA tools, but they also noted various limitations in supporting learning. Interestingly, experimental studies that statistically measured critical thinking and cognitive learning have rarely rendered any significant gains (Johnson et al., 2010; Mendenhall, 2010; Razon et al., 2012). Optimistically, the authors believe that the extent to which collaboration is achieved invariably depends on how the instructor designs and implements the collaborative activity. As Novak et al. (2012) stated in their research, students should be provided with substantial instructional support during any collaborative learning activity to maximize the learning benefits of the SA tools.

Another issue raised in this study is an unequal contribution and participation among all the collaborators, which is fairly common in collaborative writing activities afforded by social technologies (Arnold, Ducate, \& Kost, 2009). Motivation is certainly one major contributing factor to learners' participation (Xie \& Ke, 2011). Apart from this, research on collaborative learning suggests some other factors attributable to the differences in learners' degree of participation such as learners' learning styles and their personalities (Durán, 2011; Yadegaridehkordi et al., 2013). It is largely unclear why the levels of participation are so different among students in this particular study, and there is a need for future research investigating how to optimize student participation in SA-supported collaborative learning activities.

\section{Conclusion}

In this study, student learning experiences using SA tools in three collaborative learning activities were examined. These three practices were effective in keeping students on task and stimulating them to learn. With slightly different purposes, all these activities supported with SA tools facilitated collaborative learning by allowing peer-commenting and annotating efforts. Educators who are interested in using SA tools in teaching and learning may gain insights on how to design SA-supported learning activities from this study. This study suggests the need to understand how to support prolonged discussion and promote critical thinking and meta-cognitive learning using SA tools. Questions such as in what ways, under which conditions, and within which context these SA tools could be optimally utilized to promote deeper learning should be addressed in future research. Besides conducting robust experimental research, researchers may use a design-based research approach to observe instructional activities over a longer period of time, and examine the corresponding effects of the student learning process. Additionally, more studies need to be done to provide insight on how to motivate the laggards and ensure an appropriate amount of participation across learners of all levels. 


\section{Reference}

Arnold, N., Ducate, L., \& Kost, C. (2009). Collaborative writing in wikis: Insights from culture projects in intermediate German classes. In L. Lomicka \& G. Lord (Eds.), The Next Generation: Social Networking and Online Collaboration in Foreign Language Learning (pp. 115144). San Marcos, TX : CALICO.

Durán, E. A. (2011). Personalised collaborative skills for student models. Interactive Learning Environments, 19(2), 143-162.

Glover, I., Xu, Z., \& Hardaker, G. (2007). Online annotation - Research and practices. Computers \& Education, 49(4), $1308-1320$. doi: $10.1016 /$ j.compedu.2006.02.006

Hwang, W., Wang, C., \& Sharples, M. (2007). A study of multimedia annotation of Web-based materials. Computers and Education, 48(4), 680-699. doi: 10.1016/ j.compedu.2005.04.020

Johnson, T. E., Archibald, T. N., \& Tenenbaum, G. (2010). Individual and team annotation effects on students' reading comprehension, critical thinking, and meta-cognitive skills. Computers in Human Behavior, 26, 1496-1507. doi: 10.1016/j.chb.2010.05.014

Kawasaki, Y., Sasaki, H., Yamaguchi, H., \& Yamaguchi, Y. (2008). Effectiveness of highlighting as a prompt in text reading on a computer monitor. Paper presented at the Proceedings of the 8 th WSEAS International Conference on Multimedia systems and signal processing, Hangzhou, China.

Mendenhall, A. T. E. (2010). Fostering the development of critical thinking skills, and reading comprehension of undergraduates using a Web 2.0 tool coupled with a learning system. Interactive Learning Environments, 18(3), 263-276. doi: $10.1080 / 10494820.2010 .500537$
Merrill, M.D., \& Gilbert, C.G. (2008). Effective peer interaction in a problemcentered instructional strategy. Distance Education, 29, 199-207.

Nokelainen, P., Miettinen, M., Kurhila, J., Flor, eacute, en, P., \& Tirri, H. (2005). A shared document-based annotation tool to support learner-centred collaborative learning. British Journal of Educational Technology, 36(5), 757-770. doi: 10.1111/ j.1467-8535.2005.00474.x

Novak, E., Razzouk, R., \& Johnson, T. E. (2012). The educational use of social annotation tools in higher education: A literature review. The Internet and Higher Education, 15(1), 39-49. doi: 10.1016/ j.iheduc.2011.09.002

Razon, S., Turner, J., Johnson, T. E., Arsal, G., \& Tenenbaum, G. (2012). Effects of a collaborative annotation method on students' learning and learning-related motivation and affect. Computers in Human Behavior, 28(2), 350-359. doi: 10.1016/j.chb.2011.10.004

Sakar, A., \& Ercetin, G. (2005). Effectiveness of hypermedia annotations for foreign language reading. Journal of Computer Assisted Learning, 21(1), 28-38. doi: 10.1111/j.1365-2729.2005.00108.x

Samuel, R. D., Kim, C., \& Johnson, T. E. (2011). A Study of a Social Annotation Modeling Learning System. Journal of Educational Computing Research, 45(1), 117-137.

Sweller, J. (1988). Cognitive load during problem solving: Effects on learning. Cognitive Science, 12 (2), 257-285.

Su, A. Y. S., Yang, S. J. H., Hwang, W., \& Zhang, J. (2010). A Web 2.0-based collaborative annotation system for enhancing knowledge sharing in collaborative learning environments. Computers \& Education, 55(2), 752-766. doi: 10.1016/j.compedu.2010.03.008 van der Pol, J., van den Berg, B. A. M., 
Admiraal, W. F., \& Simons, P. R. J. (2008). The nature, reception, and use of online peer feedback in higher education. Computers \& Education, 51(4), 1804-1817. doi: $10.1016 /$ j.compedu.2008.06.001

Wolfe, J. (2002). Annotation technologies: A software and research review. Computers \& Composition, 19(4), 471.

Wolfe, J. (2008). Annotations and the collaborative digital library: Effects of an aligned annotation interface on student argumentation and reading strategies. International Journal of ComputerSupported Collaborative Learning, 3(2), 141-164. doi: 10.1007/s11412-008-9040-x

Xie, K., \& Ke, F. (2011). The role of students' motivation in peer-moderated asynchronous online discussions. British Journal Of Educational Technology, 42(6), 916-930. doi:10.1111/j.14678535.2010.01140.x

Yadegaridehkordi, E., Iahad, N. A., \& Ahmad, N. (2013). Collaborative Learning Tools in Higher Education: Literature Review (2007-2012). Australian Journal Of Basic \& Applied Sciences, 7(8), 285-296. 


\section{Contact the Authors}

\section{Tian Luo}

Ohio University

Email: luot@ohio.edu

\section{Fei Gao}

Bowling Green State University

Email: gaof@bgsu.edu

\section{Kathryn S Hoff}

Bowling Green State University

Email:khoff@bgsu.edu 